\title{
Non-Local Means Denoising
}

\author{
Antoni Buades ${ }^{1}$, Bartomeu Coll ${ }^{2}$, Jean-Michel Morel ${ }^{3}$ \\ ${ }^{1}$ CNRS-Paris Descartes, France (toni.buades@uib.es) \\ ${ }^{2}$ Universitat Illes Balears, Spain (tomeu.coll@uib.es) \\ ${ }^{3}$ CMLA, ENS Cachan, France (morel@cmla.ens-cachan.fr) \\ Communicated by Guoshen Yu Demo edited by Miguel Colom
}

\begin{abstract}
We present in this paper a new denoising method called non-local means. The method is based on a simple principle: replacing the color of a pixel with an average of the colors of similar pixels. But the most similar pixels to a given pixel have no reason to be close at all. It is therefore licit to scan a vast portion of the image in search of all the pixels that really resemble the pixel one wants to denoise. The paper presents two implementations of the method and displays some results.
\end{abstract}

\section{Source Code}

The source code (ANSI C), its documentation, and the online demo are accessible at the IPOL web page of this article ${ }^{1}$. Some of the files use algorithms possibly linked to patent [3]. These files are made available for the exclusive aim of serving as scientific tool to verify the soundness and completeness of the algorithm description. Compilation, execution and redistribution of these files may violate exclusive patents rights in certain countries. The situation being different for every country and changing over time, it is your responsibility to determine which patent rights restrictions apply to you before you compile, use, modify, or redistribute these files. The rest of files are distributed under GPL license. A C/C++ implementation is provided. Please see the readme file or the online documentation for details.

Keywords: nlmeans; non-local means; non-local; denoising

\section{Introduction}

In any digital image, the measurement of the three observed color values at each pixel is subject to some perturbations. These perturbations are due to the random nature of the photon counting process in each sensor. The noise can be amplified by digital corrections of the camera or by any image processing software. For example, tools removing blur from images or increasing the contrast enhance the noise.

\footnotetext{
${ }^{1}$ https://doi.org/10.5201/ipol.2011.bcm_nlm
} 
The principle of the first denoising methods was quite simple: replacing the color of a pixel with an average of the colors of nearby pixels. The variance law in probability theory ensures that if nine pixels are averaged, the noise standard deviation of the average is divided by three. Thus, if we can find for each pixel nine other pixels in the image with the same color (up to the fluctuations due to noise) one can divide the noise by three (and by four with 16 similar pixels, and so on). This looks promising, but where can these similar pixels be found?

The most similar pixels to a given pixel have no reason to be close at all. Think of the periodic patterns, or the elongated edges which appear in most images. It is therefore licit to scan a vast portion of the image in search of all the pixels that really resemble the pixel one wants to denoise. Denoising is then done by computing the average color of these most resembling pixels. The resemblance is evaluated by comparing a whole window around each pixel, and not just the color. This new filter is called non-local means [1,2] and it writes

$$
N L u(p)=\frac{1}{C(p)} \int f(d(B(p), B(q)) u(q) d q
$$

where $d(B(p), B(q))$ is an Euclidean distance between image patches centered respectively at $p$ and $q, f$ is a decreasing function and $C(p)$ is the normalizing factor.

Since the search for similar pixels will be made in a larger neighborhood, but still locally, the name "non-local" is somewhat misleading. In fact Fourier methods for example are by far more nonlocal than NL-means. Nevertheless, the term is by now sanctified by usage and for that reason we shall keep it. The term "semi-local" would have been more appropriate, though. The implementation of the current on line demo ${ }^{2}$ is based on a patch version of the original NL-means. This version is based on a simple observation. When computing the Euclidean distance $d(B(p), B(q))$, all pixels in the patch $B(p)$ have the same importance, and therefore the weight $f(d(B(p), B(q))$ can be used to denoise all pixels in the patch $B(p)$ and not only $p$.

For completeness, we shall give both the original (pixelwise) presentation, and the patchwise presentation of the same algorithm, which is somewhat more elegant.

\section{Pixelwise Implementation}

The denoising of a color image $u=\left(u_{1}, u_{2}, u_{3}\right)$ and a certain pixel $p$ writes

$$
\hat{u}_{i}(p)=\frac{1}{C(p)} \sum_{q \in B(p, r)} u_{i}(q) w(p, q), \quad C(p)=\sum_{q \in B(p, r)} w(p, q),
$$

where $i=1,2,3$ and $B(p, r)$ indicates a neighborhood centered at $p$ and with size $(2 r+1) \times(2 r+1)$ pixels. This research zone is limited to a square neighborhood of fixed size because of computation restrictions. This is a $21 \times 21$ window for small and moderate values of $\sigma$. The size of the research window is increased to $35 \times 35$ for large values of $\sigma$ due to the necessity of finding more similar pixels to reduce further the noise.

The weight $w(p, q)$ depends on the squared Euclidean distance $d^{2}=d^{2}(B(p, f), B(q, f))$ of the $(2 f+1) \times(2 f+1)$ color patches centered respectively at $p$ and $q$

$$
d^{2}(B(p, f), B(q, f))=\frac{1}{3(2 f+1)^{2}} \sum_{i=1}^{3} \sum_{j \in B(0, f)}\left(u_{i}(p+j)-u_{i}(q+j)\right)^{2} .
$$

\footnotetext{
${ }^{2}$ https://doi.org/10.5201/ipol.2011.bcm_nlm
} 
That is, each pixel value is restored as an average of the most resembling pixels, where this resemblance is computed in the color image. So for each pixel, each channel value is the result of the average of the same pixels.

We use an exponential kernel in order to compute the weights $w(p, q)$

$$
w(p, q)=e^{-\frac{\max \left(d^{2}-2 \sigma^{2}, 0.0\right)}{h^{2}}},
$$

where $\sigma$ denotes the standard deviation of the noise and $h$ is the filtering parameter set depending on the value of $\sigma$. The weight function is set in order to average similar patches up to noise. That is, patches with square distances smaller than $2 \sigma^{2}$ are set to 1 , while larger distances decrease rapidly accordingly to the exponential kernel.

The weight of the reference pixel $p$ in the average is set to the maximum of the weights in the neighborhood $B(p, r)$. This setting avoids the excessive weighting of the reference point in the average. Otherwise, $w(p, p)$ should be equal to 1 and a larger value of $h$ would be necessary to ensure the noise reduction. By applying the above averaging procedure we recover a denoised value at each pixel $p$.

\section{Patchwise Implementation}

The denoising of a color image $u=\left(u_{1}, u_{2}, u_{3}\right)$ and a certain patch $B=B(p, f)$ (centered at $p$ and with size $(2 f+1) \times(2 f+1))$ writes

$$
\hat{B}_{i}=\frac{1}{C} \sum_{Q=Q(q, f) \in B(p, r)} u_{i}(Q) w(B, Q), \quad C=\sum_{Q=Q(q, f) \in B(p, r)} w(B, Q),
$$

where $i=1,2,3, B(p, r)$ indicates a neighborhood centered at $p$ and with size $(2 r+1) \times(2 r+1)$ pixels and $w(B(p, f), B(q, f))$ has the same formulation than in the pixelwise implementation.

In this way, by applying the procedure for all patches in the image, we shall dispose of $N^{2}=$ $(2 f+1)^{2}$ possible estimates for each pixel. These estimates can be finally averaged at each pixel location in order to build the final denoised image

$$
\hat{u}_{i}(p)=\frac{1}{N^{2}} \sum_{Q=Q(q, f) \mid q \in B(p, f)} \hat{Q}_{i}(p) .
$$

The main difference of both versions is the gain on PSNR by the patchwise implementation, due to the larger noise reduction of the final aggregation process. Spurious noise oscillations near edges are also reduced by the final aggregation process. However, the overall quality in terms of preservation of details is not improved by the patchwise version.

\subsection{Parameters}

The size of the patch and research window depend on the value of $\sigma$. When $\sigma$ increases we need a larger patch to make patch comparison robust enough. At the same time, we need to increase the research window size to increase the noise removal capacity of the algorithm by finding more similar pixels.

The value of the filtering parameter writes $h=k \sigma$. The value of $k$ decreases as the size of the patch increases. For larger sizes, the distance of two pure noise patches concentrates more around $2 \sigma^{2}$ and therefore a smaller value of $k$ can be used for filtering.

The set of parameters used by the method, for different values of $\sigma$ are displayed in Table 1 . 
Gray

\begin{tabular}{cccc}
$\sigma$ & Comp. Patch & Res. Block & $\mathrm{h}$ \\
\hline $0<\sigma \leq 15$ & $3 \times 3$ & $21 \times 21$ & $0.40 \sigma$ \\
$15<\sigma \leq 30$ & $5 \times 5$ & $21 \times 21$ & $0.40 \sigma$ \\
$30<\sigma \leq 45$ & $7 \times 7$ & $35 \times 35$ & $0.35 \sigma$ \\
$45<\sigma \leq 75$ & $9 \times 9$ & $35 \times 35$ & $0.35 \sigma$ \\
$75<\sigma \leq 100$ & $11 \times 11$ & $35 \times 35$ & $0.30 \sigma$
\end{tabular}

Color

\begin{tabular}{cccc}
$\sigma$ & Comp. Patch & Res. Block & $\mathrm{h}$ \\
\hline $0<\sigma \leq 25$ & $3 \times 3$ & $21 \times 21$ & $0.55 \sigma$ \\
$25<\sigma \leq 55$ & $5 \times 5$ & $35 \times 35$ & $0.40 \sigma$ \\
$55<\sigma \leq 100$ & $7 \times 7$ & $35 \times 35$ & $0.35 \sigma$
\end{tabular}

Table 1: Parameters of the patchwise implementation of the method.

\section{Examples}

The example in Figure 1 illustrates how the NLmeans algorithm is able to remove the noise while keeping the fine structures and details.
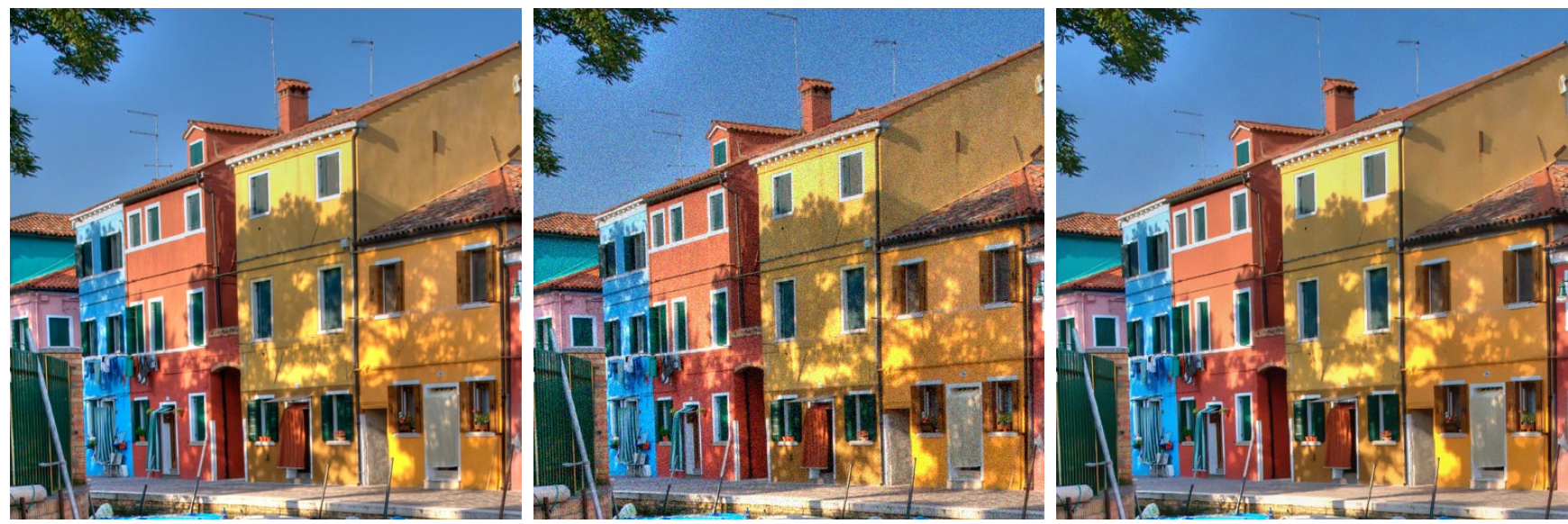

Figure 1: Example of NLmeans results. From left to right: original image, noisy image $(\sigma=15)$, denoised image.

\section{Image Credits}

CC-BY-NC-SA Herv Bry, Flickr³.

\section{References}

[1] A. Buades, B. Coll, J.M. Morel, "A review of image denoising methods, with a new one", Multiscale Modeling and Simulation, Vol. 4 (2), pp: 490-530, 2006. http://dx.doi.org/10.1137/ 040616024

[2] A. Buades, B. Coll, J.M. Morel, "A non local algorithm for image denoising", IEEE Computer Vision and Pattern Recognition 2005, Vol 2, pp: 60-65, 2005. http://dx.doi.org/10.1109/ CVPR. 2005.38

\footnotetext{
${ }^{3}$ http://www.flickr.com/photos/setaou/2162752903/
} 
[3] A. Buades, B. Coll, J.M. Morel, "Image data processing method by reducing image noise, and camera integrating means for implementing said method", EP Patent 1,749,278 (Feb. 7), 2007. 